\title{
Crise hídrica em São Paulo em 2014: seca e desmatamento
}

\author{
Jose Antonio Marengo \\ Cemaden \\ Lincoln Muniz Alves \\ CCST-Inpe
}

p. $485-494$

\section{revista}

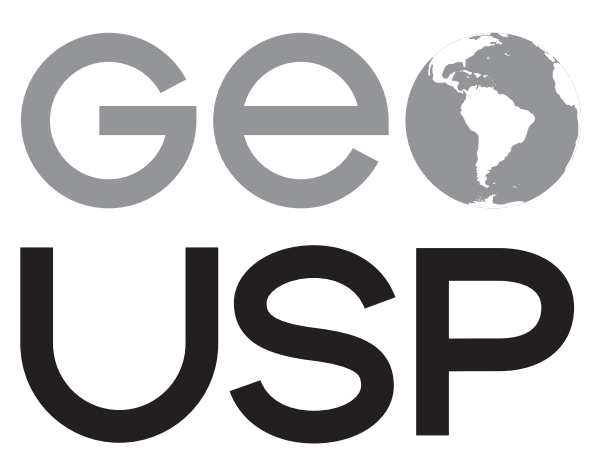

espaço e tempo

Volume $19 \cdot n^{\circ} 3(2015)$
Como citar este artigo:

MARENGO, J. A.; ALVES, L. M. Crise hídrica em São Paulo em 2014: seca e desmatamento. Geousp - Espaço e Tempo (Online), v. 19, n. 3, p. 485-494, mês. 2016. ISSN 2179-0892.

Disponível em: URL: http://www.revistas.usp.br/ geousp/article/view/100879. DOI: http://dx.doi. org/10.11606/issn.2179-0892.geousp.2015.100879.

\section{(c) (1) $(9$}

Este artigo está licenciado sob a Creative Commons Attribution 4.0 License. 


\title{
Crise hídrica em São Paulo em 2014: seca e desmatamento
}

\section{Resumo}

Este estudo traz uma análise hidrometeorológica da estação chuvosa 2013-2014 na região Sudeste do Brasil com o objetivo de diagnosticar a seca e seu impacto na disponibilidade hídrica do estado de São Paulo. Discutem-se os totais acumulados da precipitação, os principais sistemas meteorológicos atuantes durante o período chuvoso (dezembro-fevereiro) e o papel da floresta Amazônica como uma das fontes de umidade para o Brasil Central e as regiões Sul e Sudeste. Verificou-se que os totais acumulados foram significativamente abaixo dos valores médios, com desvios negativos em torno de $62 \%$, temperaturas $2,5^{\circ} \mathrm{C}$ acima da média e aumento no consumo de água. Conjuntamente, esses fatores geraram uma crise hídrica sem precedentes desde 1960.

Palavras-chave: Seca. São Paulo. Cantareira. Desmatamento. Amazônia.

\section{Water crisis in São Paulo in 2014: drought and deforestation}

\begin{abstract}
This study shows an hydrometeorological analyses of the 2013-2014 rainy season in Southeastern Brazil, in order to assess the magnitude and extension of the drought and water crises that affect the State of Sao Paulo during summer of 2014. For that we analyze mean rainfall and anomalies during the peak rainfall months (December-February). In particular, rainfall was well below normal from December 2013 to February 2014. We investigate the physical causes of this lack of rain in the region, in the context of rain producing mechanisms in the region, including the role of the Amazon tropical rainforest as one of the sources or moisture for rainfall in Southern and Southeastern Brazil. Rainfall analyses showed that summertime accumulated were about $62 \%$ below normal in 2014 , and together with a $2,5^{\circ} \mathrm{C}$ warmer summer and increase in water use, all of these generated an unprecedented water crisis since 1960.
\end{abstract}

Keywords: Drought. Sao Paulo. Cantareira. Deforestation. Amazonia. 


\section{Introdução}

As grandes secas são os desastres naturais mais custosos, superando outros desastres naturais, tais como, inundações, furacões, terremotos e tsunamis. A Organização das Nações Unidas para a Alimentação e a Agricultura (FAO) afirmam que, em geral, o fenômeno da seca gera perdas anuais na faixa de US\$ 6 e 8 bilhões. Entre 1900 e 2013, mais de 11 milhões de pessoas morreram por causa da falta d'água e 2 bilhões tiveram suas vidas transtornadas pelo fenômeno. Acumulam-se evidências de que o clima está mudando em diversas partes do planeta. Um exemplo de conhecimento recente é o fato de que a última década concentrou os 12 anos mais quentes desde que as medições começaram a ser realizadas, em 1850.

No Brasil são vários os exemplos de extremos climáticos: secas intensas na Amazônia em 2005 e 2010 e enchentes nos anos de 2009, 2012, 2014 e 2015; seca no semiárido no Nordeste desde 2013; seca e déficit hídrico no Sudeste em 2014; enchentes em Rondônia e Acre em 2014. $\mathrm{Na}$ escala de dias, destacam-se as chuvas intensas, os deslizamentos de terra e as enchentes no Vale do Itajaí em 2008, nos estados de Alagoas e Pernambuco em 2010, na região serrana do Rio de Janeiro em 2011, e em Salvador em 2015, contabilizando, juntos, mais de 2.000 mortes.

A Região Metropolitana de São Paulo (RMSP) está vivenciando uma das maiores secas da sua história. A combinação de baixos índices pluviométricos durante o verão 2014 e 2015 e um grande crescimento da demanda de água, assim como a ausência de um planejamento minimamente adequado para o gerenciamento dos recursos hídricos (e sem contar a parcela de culpa que pode ser atribuída à ausência de consciência coletiva dos consumidores brasileiros para o uso racional da água), tem gerado o que chamamos de "crise hídrica", uma crise já anunciada, pois enfrentamos situação semelhante durante a "crise do apagão" durante a seca de 2001-2002. Os baixos totais acumulados de chuva sobre a região da Cantareira, ao nordeste da RMSP afetaram significativamente a disponibilidade hídrica dos reservatórios do Sistema Cantareira, localizado na divisa entre os estados de São Paulo e Minas Gerais. $\bigcirc$ Cantareira é o principal sistema de abastecimento de São Paulo, fornecendo água a 6 milhões de habitantes na região metropolitana. $\bigcirc$ sistema também é responsável pelo abastecimento de uma população de 5 milhões de pessoas nas bacias dos rios Piracicaba, Capivari e Jundiaí.

Como consequência grave, a população vem sofrendo com o abastecimento de água em grande parte da RMSP e em diversas cidades do estado de São Paulo, sendo o racionamento de água parte da realidade atual do paulistano. Uma seca dessa magnitude, que afeta os níveis dos mananciais transformando-se em um grave problema social, é precisamente o tipo de fenômeno climático extremo projetado entre diversos impactos das mudanças climáticas já indicados nos relatórios do Intergovernmental Panel on Climate Change (IPCC). Em períodos de seca, o calor mais intenso pode aumentar a evaporação, o déficit hídrico e gerar situações de estresse hídrico, como aqueles observados em 2001 (Cavalcanti; Kousky, 2001) e mais recentemente em 2014 (Nobre et al., 2015). Outro efeito da seca foi o aumento do número de focos de queimadas. A seca também teve impactos socioeconômicos, principalmente nas áreas que exploram o turismo às margens de rios e represas.

Muitas são as especulações sobre as causas desta seca histórica: algumas sugerem uma redução do fluxo da umidade da Amazônia associada a variações na circulação atmosfera regional ou global; outras atribuem a seca ao desmatamento da Amazônia e da mata atlântica; e outras ainda as mudanças climáticas globais. Em verdade, trata-se de anomalias na circulação de verão que afetam o padrão de chuvas na região Sudeste do Brasil, modificando o transporte de umidade que vem da Amazônia, as brisas marítimas que do Oceano Atlântico Sul e a ação 
dos sistemas frontais que vêm do sul do Brasil. Em geral, pode-se dizer que a crise hídrica foi gerada por uma conjunção de fatores, entre eles, a falta de gerenciamento dos recursos hídricos, agravada pela escassez de chuva, como observado em 2001 e agora em 2014-2015.

Embora seja prematuro e difícil estabelecer uma relação direta de causa e efeito entre os fatores climáticos e a crise hídrica na RMSP neste artigo, faremos uma discussão sobre as possíveis causas da seca de 2014 e as suas prováveis conexões com o desmatamento na Amazônia e variações sazonais na circulação regional da atmosfera, bem como os impactos observados.

\section{Situação meteorológica e hidrológica no Sudeste do Brasil durante o verão de 2014}

Climatologicamente, as precipitações na região apresentam um ciclo anual bem definido, com volumes máximos nos meses de dezembro a fevereiro (verão) e valores mínimos durante o período junho-agosto (inverno). Os sistemas meteorológicos atuantes na região Sudeste do Brasil são diversos, com origens e influências também diversas. Daí a complexidade de se atribuir uma causa direta a seca de 2014.

Em geral, o regime de chuvas nesta região é influenciado pela Zona de Convergência do Atlântico Sul (ZCAS), as frentes frias, o Jato de Baixos Níveis (JBN) que transporta umidade da Amazônia para o Sul e o Sudeste do Brasil (Figura 1) e as frentes frias que vem o sul, gerando condições termodinâmicas de forte instabilidade atmosférica (pancadas de chuva - grande volume de chuva em um curto período de tempo), entre outros. Tais sistemas tem um papel importante no regime de chuvas da região Sudeste do Brasil no período de verão.

Figura 1 - Diagrama esquemático dos elementos relevantes ao transporte de umidade na América do Sul ao leste dos Andes América pelo JBN (Flecha verde mostra o transporte de umidade desde Amazônia/Oceano Atlântico Sul, respectivamente)

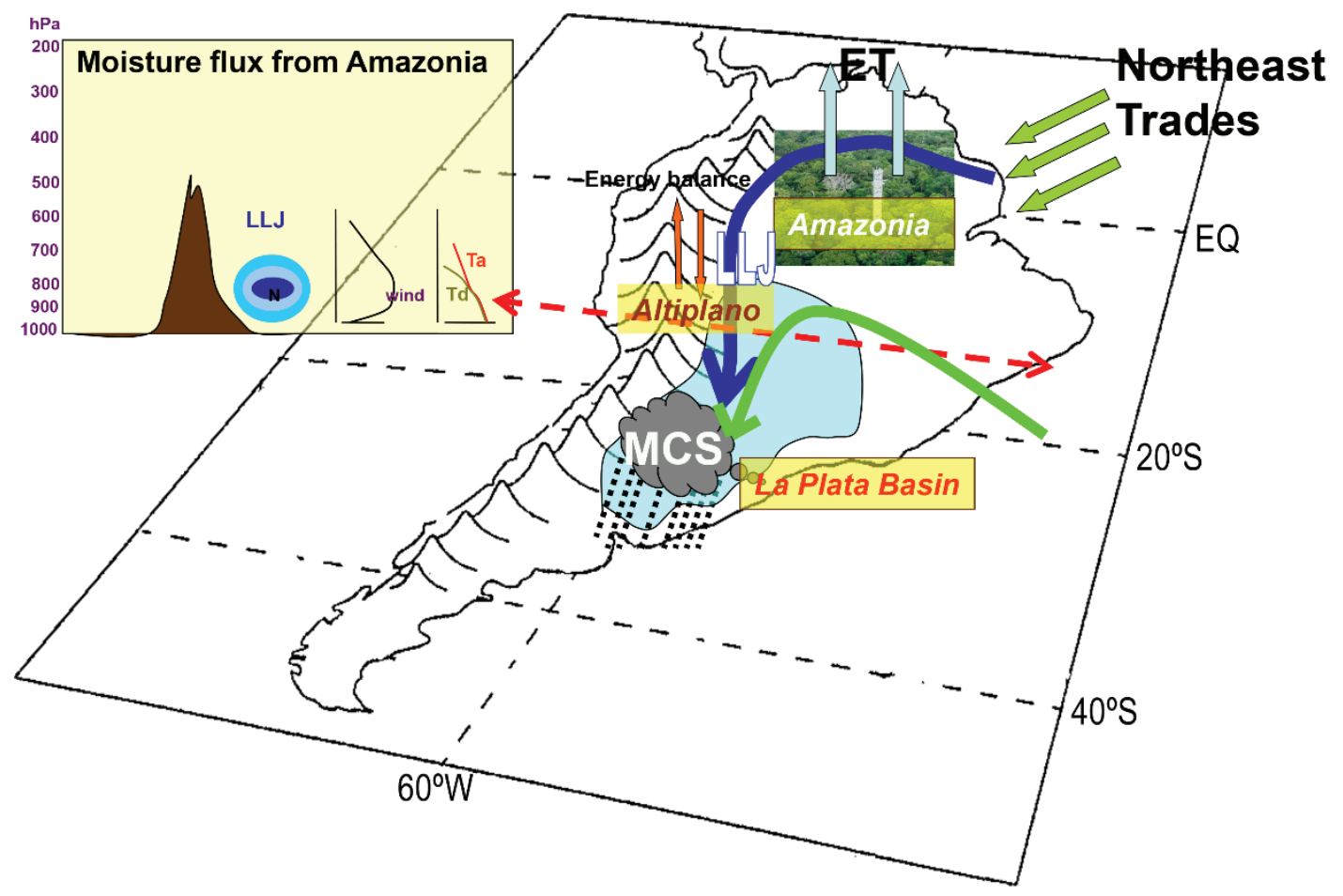

fontes: Marengo et al. (2004) e Vera et al. (2006). 
Estudos desenvolvidos pelo Cemaden e pelo Inpe indicam que a principal causa da falta de chuva no verão 2013-2014 (Figura 2) foi um intenso, persistente e anômalo sistema de alta pressão atmosférica que, inibindo as correntes ascendentes de ar, dificultou a ocorrência das típicas pancadas de chuva. No mês de dezembro de 2013, o fluxo de umidade alimentou a convecção sobre a ZCAS afetando os estados de Rondônia e Acre e ficando estacionada sobre a bacia do Rio Doce, gerando as chuvas e enchentes mais intensas das últimas décadas sobre estas regiões no estado de Espírito Santo (Marengo et al., 2014).

Figura 2 - Mapas mensais de anomalia de precipitação $(\mathrm{mm})$ sobrepostos aos campos de anomalias de circulação a $850 \mathrm{hPa}$ para (a) dezembro 2013, (b) janeiro 2014 e (c) fevereiro de 2014. As anomalias de circulação são do NCEP e relativas à média de 1980-2010. As anomalias de precipitação são do CPTEC/Inpe e relativas à média de 1961-1990. O quadrado indica a localização do estado de São Paulo
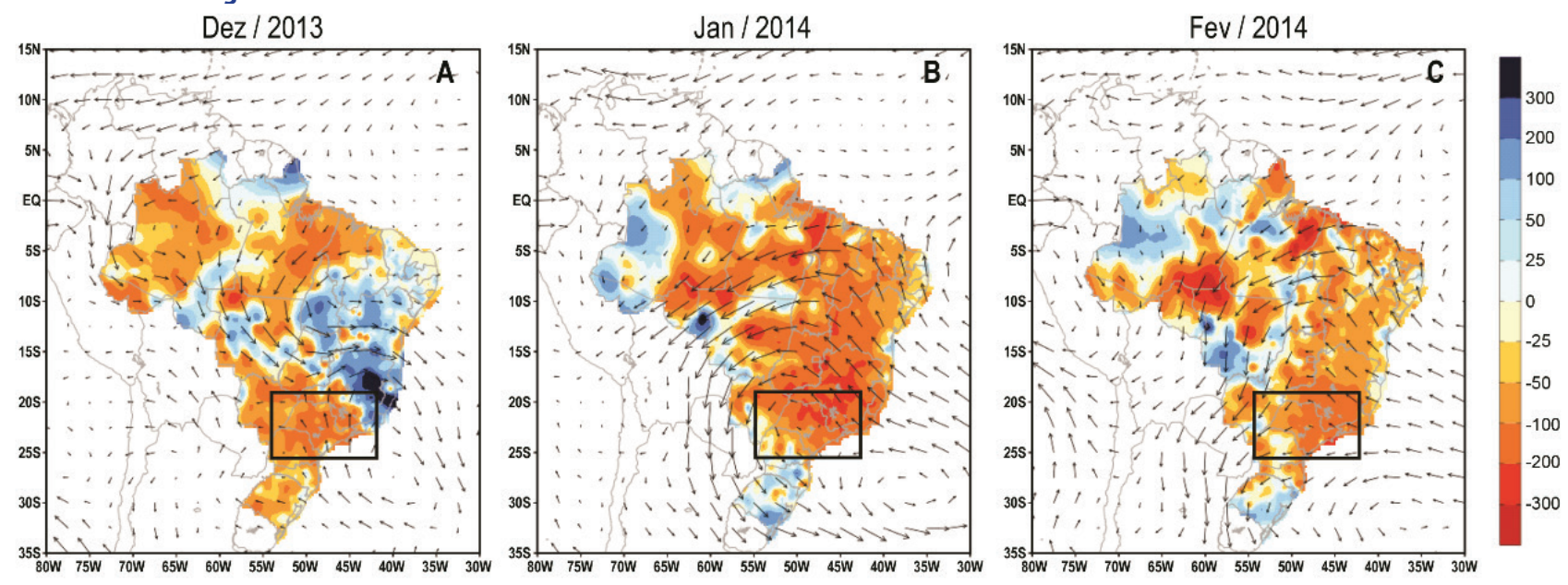

fonte: CPTEC/Inpe.

A área de alta pressão, identificada pela circulação anti-horária sobre o Sudeste do Brasil nas Figuras la-c, não permitiu a atuação da ZCAS nem a passagem/desenvolvimento das frentes frias e do transporte de umidade da Amazônia pelo JBN para região de São Paulo (quadrado nos mapas da Figura 2), normalmente responsáveis pelos maiores acumulados de chuva ao longo da estação chuvosa. De fato, a umidade transportada pelo JBN da Amazônia não penetrou no Sudeste do Brasil e foi desviada para o oeste da Amazônia, gerando as chuvas intensas e enchentes nos estados de Rondônia e Acre no verão de 2014 (Espinoza et al., 2014). Informações publicadas pelas agências do governo federal e estadual estimam em aproximadamente $\mathrm{R} \$ 203$ milhões o prejuízo causado ao estado do Acre pelas cheias que afetam os rios amazônicos desde o início de fevereiro. A Federação do Comércio do Estado acredita que os efeitos vão gerar impacto na economia nos próximos três anos. Em março, houve uma queda de $75 \%$ no imposto sobre circulação de mercadorias. Além dos prejuízos diretos com as enchentes, sobretudo no rio Acre, o estado sofre as consequências da interdição da BR-364, única ligação rodoviária com o restante do país. 
Esse sistema anômalo de alta pressão, consequência de um bloqueio atmosférico teve uma duração de aproximadamente 45 dias, de janeiro a fevereiro de 2014, sendo os índices de chuva sobre a região da Cantareira muito reduzidos (Figura 3). $\bigcirc$ bloqueio atmosférico que se iniciou a começos do mês de janeiro 2014 permaneceu até meados do mês de fevereiro, quando uma frente fria mais intensa conseguiu deslocar o sistema de alta pressão e afastá-lo do continente. Os anticiclones de bloqueio ocorrem normalmente nas latitudes médias particularmente sobre o Oceano Pacífico (Oliveira, 2011) e duram entre 7-8 dias e, em casos muito raros, podem chegar até 15 dias (Oliveira; Carvalho; Ambrizzi, 2014).

Figura 3 - Séries de tempo de chuva na região da Cantareira, desde outubro de 2012 até junho de 2015. A média climatológica é representada pelas barras cinzas (1961-2010), e as em azul são as observações durante o período

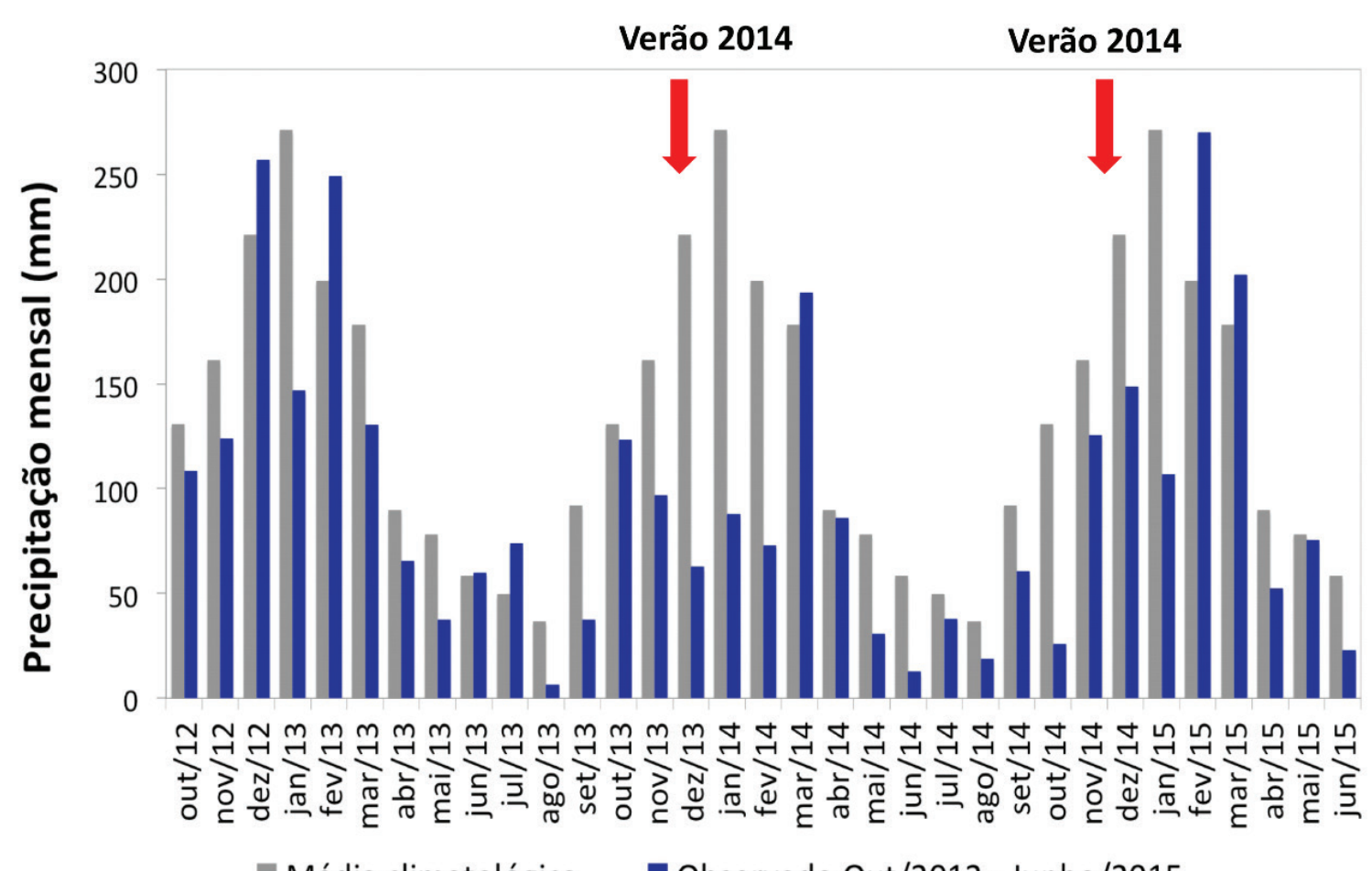

Média climatológica

- Observado Out/2012 - Junho/2015

fonte: Cemaden, 2015, p. 2.

A média histórica 1961-1990 sobre a região da Cantareira durante janeiro é 250 mm e durante o verão de 2014 foi apenas de $65 \mathrm{~mm}$. As chuvas durante 2014 foram as mais baixas desde 1961 na Cantareira, sendo a climatologia de verão (1961-1990) 249.9 mm e o acumulado durante DJF 2014 de $94.5 \mathrm{~mm}$. Consequentemente, as vazões anuais médias de entrada no sistema foram reduzidas e ficaram em torno de $44.1 \mathrm{~m}^{3} / \mathrm{s}$ (no período de 1930-2013). Em 1953, foi de $24.6 \mathrm{~m}^{3} / \mathrm{s}$ e, em 2014, chegou a $11.3 \mathrm{~m}^{3} / \mathrm{s}$. As vazões de entrada no sistema em 2014 foram as menores desde 1930. Segundo Nobre et al. (2015) e Obregón, Marengo e Nobre (2014), os totais acumulados de chuva sobre o Sistema Cantareira tem diminuído durante as últimas décadas, particularmente desde 1990. 


\section{Transporte de umidade atmosférica da Amazônia para o Sul e o Sudeste do Brasil e a seca de 2014 em São Paulo}

O JBN é um dos componentes da Monção da América do Sul e este transporte de umidade para o Sul e Sudeste do Brasil, concentrado no verão e outono alimenta os sistemas convectivos que produzem chuvas nestas regiões e na bacia do Prata (Figura 1). Vários estudos têm analisado o JBN e o transporte de umidade para a bacia do Prata (Vera et al., 2006; Marengo et al., 2004, 2012; Arraut et al., 2012, entre outros), e as principais conclusões são que o JBN contribui significativamente para as chuvas de verão em São Paulo, entretanto, não é o único ou principal sistema.

O conteúdo de umidade transportado pelo JBN vai depender dos padrões de circulação geral da atmosfera e dos gradientes de temperatura da superfície no Oceano Atlântico tropical. A floresta tropical também contribuí para acrescentar o conteúdo de umidade através de uma intensa evapotranspiração e reciclagem de umidade. $O$ conceito de reciclagem de precipitação refere-se ao mecanismo de retroalimentação ou feedback entre a superfície e a atmosfera onde a evapotranspiração local contribui, significativamente, na precipitação total sobre uma região. Em outras palavras, a reciclagem de precipitação pode ser definida como a quantidade de água que evapotranspira da superfície terrestre em uma determinada região e retorna na forma de precipitação sobre a mesma região (Brubaker; Entekhabi; Eagleson, 1993; Eltahir; Bras, 1994; Trenberth, 1999; Rocha; Silva Correia; Morelli Fonseca, 2015).

Em termos de porcentagens de contribuição para as chuvas no Sul e Sudeste do Brasil, e apesar de todos os estudos científicos já desenvolvidos, ainda não é possível estabelecer qual é a mais importante fonte de umidade, a floresta Amazônica ou o transporte de umidade do Oceano Atlântico. Angelini et al. (2011) sugere que a maior contribuição de umidade para chuva vem dos padrões de circulação de grande escala não pela evaporação local. Por outro lado, Makarieva et al. (2013), Makarieva e Gorshkov (2007), Sheil e Murdiyarso, (2009), e Nobre (2014) sugerem que o vapor de água liberado para a atmosfera via evaporação das florestas representa um reservatório de energia potencial disponível para acelerar o fluxo do ar, e que a florestas bombeiam umidade para a atmosfera.

Uma das hipóteses sobre as causas da seca de 2014 está associada ao desmatamento da Amazônia. Experimentos numéricos sobre esse desmatamento sugerem que ele pode gerar ressecamento e aumento da temperatura, afetando o clima e hidrologia na região (Magrin et al., 2014). O trabalho de Soares e Marengo (2009) aponta que, para o futuro, num cenário de altas emissões, pode-se esperar uma intensificação do transporte de umidade da Amazônia para o Sul-Sudeste do Brasil, gerando eventos mais intensos de chuvas nessas regiões, e não um regime seco ou desértico. Mas esses impactos são de longo prazo e não devem ser considerados causa de um fenômeno na escala sazonal, como foi a seca de 2013-2014.

Além dessas projeções deve-se levar em consideração a variabilidade natural do clima, por exemplo, oscilações decadais de chuva na Amazônia, que influência períodos relativamente secos e chuvosos. Adicionalmente, estudos de Silva Dias, Dias e Carvalho (2012) e Marengo et al. (2013) tem identificado tendências de aumento na frequência dias com chuva na RMSP desde 1930 devido ao efeito de urbanização, o que contrasta com a tendência detectada de diminuição das chuvas nas últimas décadas na região da Cantareira. 


\section{Considerações finais}

Os paulistanos enfrentaram em 2014 uma crise hídrica, a pior dos últimos 80 anos. A população mudou seus hábitos e aprendeu a economizar água, passou a acompanhar diariamente as medições do nível do principal sistema de abastecimento da capital, o Cantareira, e esperou, ansiosa, pela temporada de chuvas.

Através da análise dos resultados obtidos, conclui-se que, em termos sazonais, associouse a causa da seca de 2014 à persistência de uma alta pressão sobre o sudeste da América do Sul, gerando um "bloqueio atmosférico", que impediu a entrada da umidade da Amazônia através do JBN, a formação da ZCAS e a passagem das frentes frias sobre o Sudeste do Brasil. Em outras palavras, o fenômeno da seca teve sua causa associada a variabilidade natural do tempo e do clima.

A análise temporal evidenciou que os totais acumulados de precipitação durante o verão de 2014 no Cantareira foram os mais baixos desde 1961. Importante ressaltar que o período de dados analisado é suficiente para detectar a variabilidade decadal e de longo prazo na precipitação, entretanto, curta para relacionar a mudança na precipitação com o desmatamento da Amazônia, ou seja, não há evidências observacionais de que a seca na região Sudeste é resultado direto do desmatamento da floresta Amazônica ou de reduções no transporte de umidade da Amazônia para o Sudeste devido ao desmatamento já observado.

Por outro lado, a longo prazo, experimentos de modelagem numérica na Amazônia sugerem que, nas próximas décadas, haverá diminuição das chuvas, aquecimento na Amazônia e anomalias no transporte de umidade para o sudeste da América do Sul, o que pode aumentar as chuvas intensas concentradas em poucos dias e com períodos secos, que, em alguns casos, podem se estender em duração, gerando secas mais intensas e longas, como as de 2014 .

Brasil deve se preparar como qualquer país para mais eventos climáticos extremos. Os eventos observados recentemente - falta ou excesso de chuva, impactos na agricultura e saúde e perdas econômicas bilionárias - são uma amostra bem pequena de um futuro próximo. As projeções globais do IPCC e regionais do Inpe constatam clima menos chuvoso no semiárido do Nordeste e na Amazônia e aumento do índice anual de chuvas no Sul do Brasil, assim como chuvas extremas e períodos secos longos e intensos no Sudeste e Sul do país. Isso aumenta a probabilidade de eventos extremos e desastres naturais em áreas vulneráveis. Educação é também um fator a ser considerado, pois o gerenciamento dos recursos hídricos pelos governos e a redução no desperdício de água pela população são modos de enfrentar a crise hídrica. Após os recentes extremos climáticos no Brasil, nota-se que está mudando a percepção da população sobre as mudanças climáticas. Segundo o levantamento encomendado pelo Observatório do Clima e pelo Greenpeace Brasil, 95\% dos cidadãos acham que as mudanças climáticas já estão afetando o Brasil. 
Enfim, além das mudanças climáticas relacionadas ao aquecimento global, a gestão dos recursos hídricos nas grandes cidades deve ser uma prioridade dos tomadores de decisão. Uma das principais recomendações feitas pelo IPCC para enfrentar o problema de falta de água é substituir o uso de reservatórios superficiais pelo abastecimento por aquíferos. Estudos apontam que, com temperaturas mais altas, as perdas com evaporação em reservatórios abertos podem chegar a índices de 30\% a 40\%. Talvez essa solução não seja adequada a nossa realidade, entretanto, devem-se pensar e planejar ações de médio e longo prazo, levando sempre em conta as projeções climáticas futuras, em cenário otimista ou pessimista. São necessárias ações de comunicação voltadas não só à orientação da população, apontando providências a serem efetivamente tomadas para economia de água, mas também à gravidade da situação.

\section{Referências}

ANGELINI, I. M. et al. On the coupling between vegetation and the atmosphere. Theoretical and Applied Climatology, v. 105, p. 243-261, 2011. doi: 10.1007/s00704-010-0377-5.

ARRAUT, J. M. et al. Aerial Rivers and Lakes: Looking at Large-Scale Moisture Transport and Its Relation to Amazonia and to Subtropical Rainfall in South America, Journal of Climate, v. 25, p. 543-556, 2012. doi: 10.1175/2011JCLI4189.1.

BRUBAKER, K. L.; ENTEKHABI, D.; EAGLESON, P. S. Estimation of Continental Precipitation Recycling. Journal of Climate, v. 6, p. 1077-1089, 1993.

CAVALCANTI, I. F. A.; KOUKSY, V. E. (2001) Drought in Brazil during summer and fall of 2001 and associated circulation features. Climanalise, 1: 1-10, 2001. Disponível em: <http://climanalise.cptec.inpe.br/ rclimanl/revista/pdf/criseing.pdf>. Acesso em: 13 out. 2015.

CEMADEN. CENTRO DE MONITORAMENTO E ALERTAS DE DESASTRES NATURAIS. Relatório da situação atual e projeção hidrológica para o Sistema Cantareira. São José dos Campos: Cemaden, 5 nov. 2015.

DOBROVOLSKI, R.; RATTIS, L. Water collapse in Brazil: the danger of relying on what you neglect. Natureza \& Conservação, v. 13, n. 1, p. 80-83, jan./jun. 2015. Disponível em: $<$ http://dx.doi.org/10.1016/j.ncon.2015.03.006>. Acesso em: 13 out. 2015.

ELTAHIR, E. A. B.; BRAS, R. L. Precipitation recycling in the Amazon basin. Quarterly Journal of the Royal Meteorological Society, v. 120, p. 861-880, 1994.

ESPINOZA, J. C. et al. The extreme 2014 flood in South-Western Amazon basin: The role of Tropical-Subtropical South Atlantic SST gradient. Environmental Research Letters, v. 9, n. 12, p. 124007, 2014.

MAGRIN, G. O. et al. Central and South America. In: IPCC. INTERGOVERNMENTAL PANEL ON CLIMATE CHANGE. Climate Change 2014: Impacts, Adaptation and Vulnerability. Contribution of Working Group II to the Fifth Assessment Report of the Intergovernmental Panel on Climate Change. Cambridge: Cambridge University Press, 2014. 
MAKARIEVA, A. M.; GORSHKOV, V. G. Biotic pump of atmospheric moisture as driver of the hydrological cycle on land. Hydrology and Earth System Sciences, v. 11, p. 1013-1033, 2007.

et al. Where do winds come from? A new theory on how water vapor condensation influences atmospheric pressure and dynamics. Atmospheric Chemistry and Physics, v. 13, p. 1039-1056, 2013. doi:10.5194/ acp-13-1039-2013.

MARENGO, J. A.; ESPINOZA, J. C. Extreme seasonal droughts and floods in Amazonia: causes, trends and impacts, International Journal of Climatology, 2015. doi: 10.1002/joc. 4420.

MARENGO, J. A.; VALVERDE, M. C.; OBREGON, G. O. Assessments of observed and projected changes in rainfall extremes in the Metropolitan Area of São Paulo (MASP). Climate Research, v. 57, p. 61-72, 2013.

MARENGO, J. A. et al. Tropical South America east of the Andes. [in "State of the Climate in 2014"], Bulletin of the American Meteorological Society, v. 95, p. S170-S171, 2014.

MARENGO, J. A. et al. Recent Developments on the South American monsoon System. International Journal of Climatology, v. 32, p. 1-21, 2012.

MARENGO, J. A. et al. Climatology of the low-level jet east of the Andes as derived from the NCEP-NCAR reanalyses: Characteristics and temporal variability. Journal of Climate, v. 17, p. 2261-2280, 2004.

NOBRE, A. O futuro climático da Amazônia: relatório de avaliação científica. São Paulo: ARA. Articulação Regional Amazônica, 2014. et al. The record drought and water crises of summer 2014 in Southeastern Brasil, Bullettin of American Meteorological Society, 2015. In press.

OBREGÓN, G. O.; MARENGO, J. A.; NOBRE, C. A. Rainfall and climate variability: long-term trends in the Metropolitan Area of São Paulo in the 20th century. Inter-Research - Climate Research, v. 61, n. 2, p. 93-107, 2014. doi: 10.3354/cr01241.

OLIVEIRA, F. N. M. Climatologia de bloqueios atmosféricos no hemisfério sul: observações, simulações do clima do século XX e cenários futuros de mudanças climáticas. Tese (Doutorado em Meteorologia) - Instituto de Astronomia, Geofísica e Ciências Atmosféricas, Universidade de São Paulo, São Paulo, 2011.

; CARVALHO, L. M. V.; AMBRIZZI, T. A new climatology for Southern Hemisphere blockings in the winter and the combined effect of ENSO and SAM phases. International Journal of Climatology, v. 34, p. 1676-1692, 2014. doi:10.1002/joc.3795.

ROCHA, V. M.; SILVA CORREIA, F.W.; MORELLI FONSECA, P. A. Reciclagem de precipitação na Amazônia: um estudo de revisão. Revista Brasileira de Meteorologia, v. 30, p. 59-70, 2015.

SHEIL, D.; MURDIYARSO, D. How forests attract rain: an examination of a new hypothesis. Bioscience, v. 59, p. 341-347, 2009. 
SILVA DIAS, M. A. F. et al. Changes in extreme daily rainfall for São Paulo, Brazil. Climatic Change, v. 116, p. 705-722, 2012.

SOARES, W.; MARENGO, J. A. Assessments of moisture fluxes east of the Andes in South America in a global warming scenario. International Journal of Climatology, 2009. DOI: $10.1002 /$ joc. 1800

TRENBERTH, K. E. Atmospheric Moisture Recycling: Role of Advection and Local Evaporation. Journal of Climate, v. 12, p. 1368-1381, 1999.

VERA, C. et al. The South American Low Level Jet Experiment. Bullettin of American Meteorological Society, 2006. doi: 10.1175/BAMS-87-1-63. 